\title{
Computational Modeling of Blast Induced Human Injury Biomechanics and Traumatic Brain Injury
}

X Gary $\operatorname{Tan}^{1,2^{*}}$, Andrzej J Przekwas ${ }^{2}$ and Raj K Gupta ${ }^{3}$

${ }^{1}$ CFD Research Corporation, Huntsville, AL, USA

${ }^{2}$ US Naval Research Laboratory, Washington, DC, USA

${ }^{3}$ US Army Medical Research and Materiel Command, Fort Detrick, Frederick, MD, USA

*Corresponding author: X Gary Tan, US Naval Research Laboratory, Washington, DC 20375, USA, Tel: +(1)2024041123; E-mail: gary.tan@nrl.navy.mil

Rec date: October 24, 2017; Acc date: October 28, 2017; Pub date: October 30, 2017

Copyright: @ 2017 Tan XG, et al. This is an open-access article distributed under the terms of the creative commons attribution license, which permits unrestricted use, distribution, and reproduction in any medium, provided the original author and source are credited.

\begin{abstract}
The work aims to understand the blast induced injury mechanism and facilitate the development of protection and treatment. Novel multi-scale and multi-physics computational models of coupled blast physics, whole body biodynamics and injury biomechanics are presented. Modeling components include blast wave threat characterization, anatomy-based high-fidelity human model, human body blast loading, biodynamics and body/brain biomechanics leading to primary injury, as well as the multi-physics solver suitable for high-performance computing. The coupled gas dynamics and biomechanics solutions were validated against shock tube test data. The parametric simulations of human body exposed to blasts were conducted to find biomechanical responses and brain injury mechanism.
\end{abstract}

Keywords: Blast waves; Human body; Injury biomechanics; Traumatic brain injury

\section{Introduction}

Improvised explosive devices (IEDs) have caused devastating casualties in terrorist bomb blast events, and are the main cause of traumatic brain injury (TBI). Until recently, it was not clear how a blast wave penetrates the cranium and causes the brain injury or if combat helmets protect against it. Unlike the blunt or ballistic loading, focused on a specific area of the body, the blast wave engulfs the entire human body [1]. Blast loading can be directly transmitted to head, lung, gut, groin, and extremity but the loads may also be transmitted to the brain via so-called "thoracic" or "vascular" mechanism [2].

The majority of blast injury research was conducted using human surrogate and cadaver testing, in vitro study, and analysis of clinical data, all of which are useful and necessary but lack injury scaling and prediction capability. Better understanding of the blast induced injury mechanisms can be achieved with a complementary experimental and computational modeling approach. Computational modeling of blast injury poses significant challenges as it involves several physical disciplines as well as a range of spatial and temporal scales. Most computational models of blast TBI confine their focus to modeling macroscopic biomechanics of the brain, often ignoring the presence of the elastic body, flexibility of the neck, effects of vascular and cerebral fluids and responses of the rest of body.

We presented a novel multi-physics computational approach for modeling human injury caused by the explosion blasts. We designed representative human body models, benchmark test cases for modeling blast threats and resultant injury biomechanics [1]. The multi-physics CoBi solver with domain decomposition and parallel distributed computing [3] was used for both the blast physics and human biomechanics. A high-fidelity computational fluid dynamics model was used to simulate the interaction between the human body and the blast wave generated by a representative $\mathrm{C} 4$ explosion. The computed blast pressure loads are applied to the whole-body finite element model for the injury biomechanics simulation. The coupled gas dynamics and biomechanics approach was validated against the experimental data of a shock wave interaction with a surrogate head [1] and live animal [4] in a shock tube, in which the brain pressure response is qualitatively comparable with the test data.

\section{Case Report}

The whole human body models for both blast wave dynamics and human biomechanics simulations were constructed from the Zygote human anatomical geometry. For the blast loading simulation, the human skin surface mesh is immersed in the blast scene mesh. An adaptive octree mesh extended throughout the entire blast scene and refined around the human was used to simulate blast wave dynamics and its interaction with the body. The whole-body model for the tissue/ organ biomechanics includes the detailed head (face, skull, brain and spinal cord), the neck, the skeleton, air cavities (lungs) and the tissues. A high-quality hexahedral finite element mesh was generated with the maximum element size less than three millimeters to resolve stress wavelengths as small as a few centimeters, small geometric features and material interfaces within the human body.

We employed the coupled Eulerian-Lagrangian approach for modeling the interaction between the high explosive detonation products and human body. The Eulerian-based compressible flow solver was used for modeling the expansion of detonation gaseous products and capturing the detailed shock wave interaction with human body. The simulation results of an explosion of $5 \mathrm{lb}$ of $\mathrm{C} 4$ placed $1.32 \mathrm{~m}$ above the ground with a human body located $2.3 \mathrm{~m}$ away from the explosion center are shown in Figure 1. After the initial spherical shock wave hits the ground it reflects, interacts with the primary wave and forms a strong cylindrical Mach stem wave propagating on the ground. The upper body is first loaded with the 
primary spherical wave, followed by the delayed Mach stem wave loading. The blast wave pressure loads on the entire human body are recorded for the duration of the blast event (10-20 milliseconds) and stored for consecutive human biomechanics simulations. The explicit time-stepping Lagrangian finite element method was used to simulate biomechanical response of human body organs and tissues during the pressure wave propagation within the body. Figure 1 presents the predicted pressure fields on the skin, the skeleton, the brain and spinal cord, inside the body (mid-sagittal and mid left extremity planes) during the propagation of stress waves through the body, following the free field blast impacting the body from the front. A steep fronted pressure wave traversing the brain in the forward and reflected directions can be seen.
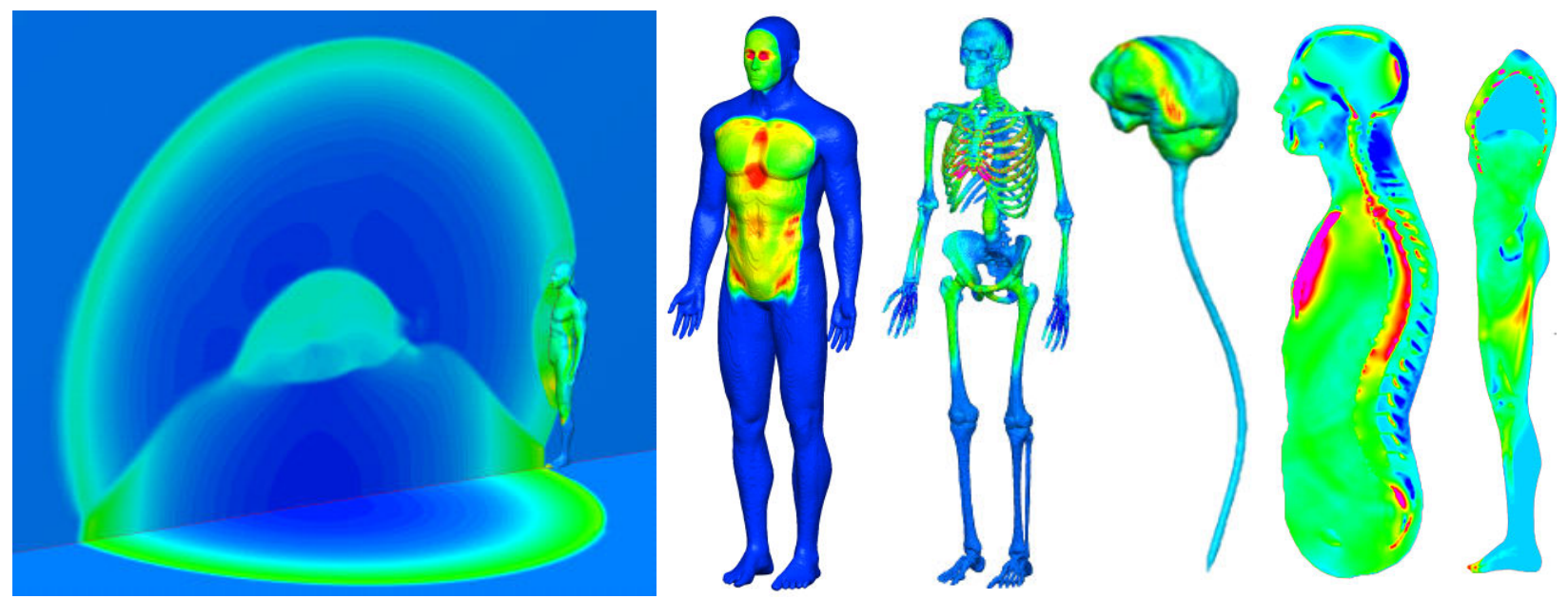

Figure 1: Simulations of human body under $5 \mathrm{lb} \mathrm{C4}$ explosion and blast induced pressures in human at different times [1].

\section{Discussion}

From the simulations we were able to confirm the coup-contrecoup phenomenon, identify the effects of the lung, and the presence of torso on the brain response. Based on both intracranial pressure and mechanical strains in the brain we found that the injury on the countercoup of brain is more severe than that on the coup site. The greater negative pressure or tensile force, occurring at the contrecoup, may be responsible for tearing of tissue and bridging vessels. In addition, the cavitation due to the negative pressure may occur and subsequent bubble collapse may cause the micro-damage in the neighboring brain tissue. The simulation results demonstrated that a stiffer material, like the skeleton, exhibits higher pressures, while the pressure in soft materials, particularly in the lung, is much lower. A relatively high level of strain appears at the interface between the skull and brain due to the low shear resistance of the brain. The maximum strain occurs in the lungs because of their softness compared to their surrounding tissue and skeleton. The parametric study also shows that the skull with higher material strength increases the intracranial pressure but mitigates both the strains and energies transmitted in the brain. These results indicate that under the same blast condition the severity of brain injury may be age and gender dependent. A detailed description and discussion of blast injury biomechanics of the whole body and of the brain was presented in the study by Tan et al. [1].

\section{Conclusion}

The whole human body model and the coupling of shock wavehuman body interaction techniques provide promising modeling capabilities of human body biomechanics under blast loading conditions. The coupled models are capable of reasonably predicting the blast loading on the human and the resulting human body/brain biomechanical response. Thus, the simulation results can be used to investigate the primary injury mechanism of a human subjected to blast loadings. They can also aid in medical diagnosis through rigorously evaluating patient specific biomechanical injury severity scores. The extension of the current investigation includes the blastinduced long-term biodynamics $[5,6]$ and the coupling of the primary brain stress and strain fields with micro-scale injury response of selected tissue structures, such as brain axonal tracks, spinal cord, cochlear, vasculature and other microstructures [7,8]. Further improvements to achieve more accurate injury prediction rely on the model with refined material differentiation and accurate constitutive laws for biological materials, along with the experiment-based tissue injury criteria.

\section{References}

1. Tan XG, Przekwas AJ, Gupta RK (2017) Computational modeling of blast wave interaction with a human body and assessment of traumatic brain injury. Shock Waves 1-16.

2. Gupta RJ, Przekwas AJ (2013) Mathematical models of blast induced TBI: Current status, challenges and prospects. Front Neurol 4: 59.

3. Kannan R, Harrand V, Tan XG, Yang HQ, Przekwas AJ (2014) Highly scalable computational algorithms on emerging parallel machine multicore architectures II: Development and implementation in the CSD and FSI contexts. J Parallel Distrib Comput 74: 2808-2817.

4. Tan XG, Przekwas, AJ, Long, JB (2013) Validations of virtual animal model for investigation of shock/blast wave TBI. In: IMECE 2013-64587, Proceedings of the ASME International Mechanical Engineering Congress and Exposition.

5. Tan XG, Przekwas AJ (2011) A computational model for articulated human body dynamics. Int J Human Factors Model Simulation 2: 85-110.

6. Tan XG, Kannan R, Przekwas AJ, Ott K, Harrigan T, et al. (2012) An enhanced articulated human body model under $\mathrm{C} 4$ blast loadings. In: IMECE 2012-89067, Proceedings of the ASME International Mechanical Engineering Congress and Exposition. 
Citation: Tan XG, Przekwas AJ, Gupta RK (2017) Computational Modeling of Blast Induced Human Injury Biomechanics and Traumatic Brain Injury. J Trauma Treat 6: 399. doi:10.4172/2167-1222.1000399

Page 3 of 3

7. Tan XG, Przekwas AJ, Gupta RK (2016) Macro-micro biomechanics finite element modeling of brain injury under concussive loadings. In IMECE2016-66218, Proceedings of the ASME International Mechanical Engineering Congress and Exposition.
8. Gupta RK, Tan XG, Somayaji MR, Przekwas AJ (2017) Multiscale modeling of blast-induced TBI mechanobiology - From body to neuron to molecule. Defence Life Science J 2: 3-13. 